\title{
Obtaining Protein Hydrolyzates By-products of Agaricus Bisporus
}

\section{Obtención de Hidrolizados Proteicos de Sub- productos de Agaricus Bisporus}

\author{
Alberto Renato Inca-Torres ${ }^{1,2}$, Anabell Urbina-Salazar ${ }^{2,3}$, Valeria Inca-Torres, \\ and Juan Bautista ${ }^{4}$ \\ ${ }^{1}$ Universidad Técnica de Ambato, Facultad de Ciencias de la Salud, Ambato, Ecuador \\ ${ }^{2}$ Universidad de Sevilla, Facultad de Farmacia, Departamento de Bioquímica y Biología \\ Molecular, Sevilla, España \\ ${ }^{3}$ Universidad Técnica de Manabí, Instituto de Ciecias Básicas, Portoviejo, Ecuador \\ ${ }^{4}$ Hospital Básico Baños, Baños, Ecuador
}

I International Congress of Science and Technology Morona Santiago-CICTMS 2020

Corresponding Author:

Alberto Renato Inca-Torres

dr.renatoinca@hotmail.com

Published: 29 August 2021

Production and Hosting by Knowledge E

(c) Alberto Renato Inca-Torres et al. This article is distributed under the terms of the Creative Commons Attribution License, which permits unrestricted use and redistribution provided that the original author and source are credited.

\section{Abstract}

The mushroom industry generates non-marketable by-products that are not used or misused, these by-products are rich in protein and other beneficial compounds for health. Currently there is great interest in products rich in protein from agro-industrial waste, in this study was used mushroom by-products to obtain protein concentrates and isolates in order to improve functional properties, using a biotechnological process based on the use of proteases, with an effective approach for the maximum recovery of the components, maintaining their quality and effectiveness. It was used 4 different proteases (Alcalasa ${ }^{\circledR}$, Flavourzyme ${ }^{\circledR}$, Papain and Bioprotease LA-450) to determine which of them is most effective, the most efficient proteases that lead to a higher hydrolysis degree is used Bioprotease LA-450 and Alcalasa ${ }^{\circledR}(6.65 \pm 0.6$ and $6.21 \pm 0.9)$ respectively, obtaining a higher amount of solubilized products $(46.3 \pm 3.6 \%$ and $41.7 \pm 3 \%$ ), as well as the Bioprotease LA-450, who presents a higher content of total proteins (51. $9 \pm 4.8$ ). This hydrolyzate, due to its composition: rich in amino acids, oligopeptides and peptides, can be used as a biofertilizer in agronomy, and as a nutraceutical or nutricosmetic in food or cosmetics.

Keywords: Agaricus bisporus, Enzymes, Protein hydrolysates, By-product.

\section{Resumen}

La industria del champiñón genera subproductos no comercializables que no se utilizan o mal utilizan, estos subproductos son ricos en proteínas y otros compuestos beneficiosos para la salud. Actualmente existe gran interés en los productos ricos en proteína provenientes de residuos agroindustriales, en este estudio se utilizó los subproductos del champiñón para obtener concentrados y aislados de proteínas con el fin de mejorar las propiedades funcionales, utilizando un proceso biotecológico basado en el uso de proteasas, con un enfoque efectivo para la recuperación máxima de los componentes, manteniendo su calidad y efectividad. Se usó 4 diferentes proteasas (Alcalasa ${ }^{\circledR}$, Flavourzyme ${ }^{\circledR}$, Papaína y Bioproteasa LA-450) para determinar cuál de ellas es la más efectiva, las proteasas más eficientes que conducen a un mayor grado de hidrólisis es cuando se utilizan la Bioproteasa LA-450 y la Alcalasa ${ }^{\circledR}(6,65 \pm$ $0,6$ y $6,21 \pm 0,9)$ respectivamente, obteniéndose mayor cantidad de productos solubilizados ( $46,3 \pm 3,6 \%$ y $41,7 \pm 3 \%$ ), así como la Bioproteasa LA-450 que presenta mayor contenido de proteínas totales $(51,9 \pm 4,8)$. Este hidrolizado debido a su composición: rico en aminoácidos, oligopéptidos y péptidos puede ser utilizado como biofertilizante en agronomía, y nutracéutico o nutricosmético en alimentación o cosmética. 
Palabras Clave: Agaricus bisporus, Enzimas, Hidrolizados proteicos, Subproducto.

\section{Introducción}

La producción de champiñones comestibles, destinados al consumo fresco o en forma de conservas representa entre el 7 y el $8 \%$ de la producción mundial (10.317.092 Tm) [1]. Este gran volumen de producto genera entre el 25 y el $30 \%$ de subproductos, que consisten en tallos y champiñones no comercializables, que no se utilizan o que han sido utilizados como alimento para animales, y como fertilizante en el campo. Este subproducto al ser rico en proteínas (23-32\% p.s.), carbohidratos y en otras sustancias que promueven la salud, como la ergotioneina y el ergosterol [2, 3], podría usarse como materia prima para obtener diversos productos con alto valor añadido, y utilizarse en las industrias químico-farmacéutica, alimentaria, cosmética o en la agronomía moderna. Debido al actual interés de utilizar subproductos agroindustriales ricos en proteínas, se han desarrollado procesos para obtener concentrados y aislados de proteínas con el fin de mejorar las propiedades funcionales, uno de estos procesos es la hidrólisis de proteínas, que puede llevarse a cabo tanto por métodos químicos (ácido o alcalino) y métodos enzimáticos. Sin embargo, la hidrólisis enzimática tiene ventajas potenciales sobre la hidrólisis química tradicional, porque se basa en el uso de enzimas bajo ciertas condiciones, como la concentración de sustrato, la relación E/S, el tiempo de incubación y las condiciones físico-químicas como temperatura, agitación, $\mathrm{pH}$, con un enfoque efectivo para la recuperación máxima de los componentes, obteniendo fracciones más pequeñas y con un valor nutricional único superior a la proteína original, manteniendo su calidad y efectividad [4]. La hidrólisis enzimática se lleva a cabo por un grupo de enzimas llamadas hidrolasas, que permiten la ruptura de enlaces peptídicos, ejerciendo un efecto catalítico hidrolizante. Las enzimas más utilizadas en este proceso son las proteasas comerciales $[5,6]$. Varios estudios en los últimos años han analizado los derivados peptídicos presentes en ciertos alimentos, obteniendo alimentos funcionales utilizados como potenciales nutracéuticos [7, 8]. Estos productos alimenticios como las bebidas proteicas que existen en el mercado son ricos en proteínas y péptidos, y son obtenidas por este método [9]. Un hidrolizado está determinado por el grado de hidrólisis y la especificidad de las enzimas, que permite determinar sus características $y$, por lo tanto, sus posibles aplicaciones [10-15]. Para este propósito, en este trabajo describimos un proceso biotecnológico para obtener hidrolizados de proteínas a partir de subproductos de la industria de los hongos comestibles basados en el uso de proteasas, debido a su bajo costo, alto valor nutricional y excelente biocompatibilidad que puede ser potencialmente útil en agronomía y en cosmética. 


\section{Materiales y Métodos}

\subsection{Preparación de la materia prima}

Se utilizaron subproductos (tallos y hongos no comercializables) de la industria de hongos (Agaricus bisporus). Los subproductos se secaron, se molieron y se tamizaron, obteniéndose harina de tallos de champiñón $(\mathrm{HTCH})$, que se utilizó como material de partida.

\subsection{Caracterización de HTCH}

El contenido de humedad, cenizas, proteínas, y fibra se determinó gravimétricamente de acuerdo con los métodos estándar de AOAC, el nitrógeno total se determinó mediante el método Kjeldahl [16] y la materia orgánica se analizó por combustión seca a $550^{\circ} \mathrm{C}$ durante $6 \mathrm{hr}[17]$.

\subsection{Proteasas utilizadas}

Se utilizaron cuatro enzimas proteolíticas para determinar su actividad contra el sustrato de proteína alimentaria (subproductos de hongos). [18-20]. En la Tabla 1 se muestran las proteasas comerciales de grado alimenticio utilizadas para la hidrólisis enzimática.

\section{Table 1}

Enzimas utilizadas en la hidrólisis.

Nombre de la proteasa comercial
Alcalasa ${ }^{\circledR} 2.5$ FG E.C.3.4.21.62 Novozymes
Flavourzyme ${ }^{\circledR}$ Novozymes
Bioproteasa LA-450 E.C.3.4.21.99 (Biocon Española S.A.)
Papaína E.C.3.4.22.2 Novozymes

Microorganismo productor/Fuente

Bacillus licheniformis

Aspergillus oryzae

Bacillus licheniformis

Papaya

\subsection{Preparación del hidrolizado de proteínas}

La HTCH (30 g) se suspendió en $300 \mathrm{~mL}$ de agua (1:10 p/v) y se digirió durante $2 \mathrm{hr}$ utilizando proteasas $(0.3 \% \mathrm{E} / \mathrm{S})$ a $\mathrm{pH} 8.5$ y $55^{\circ} \mathrm{C}$, a baja agitación (100 rpm). El pH se ajustó y se mantuvo a un $\mathrm{pH}$ constante. Después de $2 \mathrm{hr}$, el hidrolizado se calentó en un baño de agua hirviendo durante 10 min para inactivar las enzimas. El hidrolizado se centrifugó y los sobrenadantes se recogieron y almacenaron a $-20^{\circ} \mathrm{C}$. El proceso de hidrólisis se realizó como se muestra en esquema de la Figura 1. 


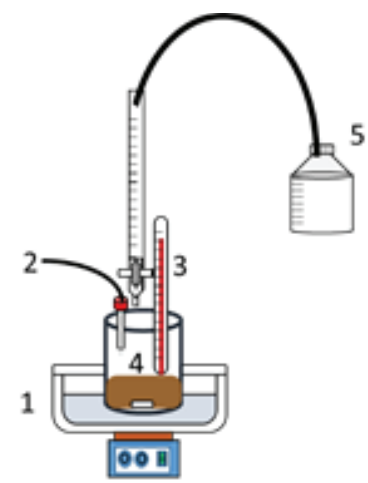

Figure 1

Esquema de ensamblaje de hidrólisis (1) Baño térmico, (2) Control de pH, (3) Control de temperatura, (4) Agitación, (5) Dosificación de $\mathrm{NaOH}$.

\subsection{Determinación del grado de hidrólisis $(\mathrm{GH})$}

El grado de hidrólisis se define como el porcentaje de enlaces peptídicos rotos con respecto a la proteína de partida [21] La ecuación que relaciona ambas variables es la siguiente (Aldler-Nissen, 1986)

$$
[22] D H=\frac{V_{B} \times N_{B}}{M_{P}} \times \frac{1}{\alpha} \times \frac{1}{h_{t o t}} \times 100,
$$

dónde: $\mathrm{V}_{B}$ : Volumen de Base añadido $(\mathrm{mL}) ; \mathrm{N}_{B}$ : Normalidad de la base; $\mathrm{M}_{P}$ : Masa Proteica (g); $\mathrm{h}_{\text {tot }}$ : Número de enlaces peptídicos de la proteína original (meqv/g); $\alpha$ : Es el grado de disociación promedio de los grupos amino liberados durante la hidrólisis.

\subsection{Determinación de peso molecular}

SDS-PAGE es el método más utilizado para analizar cuantitativamente mezclas de proteínas porque es útil para monitorear la purificación de proteínas y la separación de proteínas en función de su tamaño. Los sistemas de gel Laemmli típicos, que usan glicina en el tampón de funcionamiento, son capaces de resolver proteínas en el rango de masa molecular de $\sim 200,000$ daltons a 23000 daltons [23] y los sistemas Schägger y Von Jagow en los que la glicina se reemplaza por tricina, que permiten la resolución de péptidos tan pequeños como 500 daltons [24].

Las bandas de proteínas se fijaron y se tiñeron en Coomassie Brilliant Blue G-250. Las proteínas más pequeñas se visualizan mediante tinción con plata. Los geles se digitalizaron en el sistema de imágenes Gel Doc ${ }^{T M}$ XR+ (Bio-Rad Laboratorios, CA, EE. UU.).

\subsection{Caracterización del hidrolizado por cromatografía de exclusión}

Con el fin de analizar también oligopéptidos (<1000 Da) y tri-, dipéptidos y aminoácidos libres (no detectables por SDS-PAGE, las muestras se analizaron por cromatografía de 
exclusión molecular (HPLC). El volumen de muestra cargado ha sido de $20 \mu \mathrm{L}$ y la elución de las proteínas se ha llevado a cabo con tampón fosfato $100 \mathrm{mM} / \mathrm{NaCl} 0.25 \mathrm{M}$ a un flujo de $0.5 \mathrm{~mL} / \mathrm{min}$.

\subsection{Análisis estadístico}

El análisis de datos se realizó mediante ANOVA unidireccional seguido de las pruebas de rango múltiple de Duncan a través de SPSS Statistics 20.0 (SPSS, Inc., Chicago, IL, EE. UU.). Los experimentos se repitieron tres veces y la importancia se evaluó a $\pm 5 \%$ $(P \leq 0.05)$. Los resultados se expresaron como media \pm desviación estándar.

\section{Resultados}

\subsection{Componente básico de $\mathrm{HTCH}$}

El análisis aproximado de la $\mathrm{HTCH}$ mostró un alto contenido de proteínas (23-32\% p.s.), alto contenido de carbohidratos (56-62\%), bajo contenido de grasas (3.52\%), alto contenido de fibra cruda (17\%), 7.3\% de contenido de humedad, $92.7 \%$ correspondiente a materia seca y $8.83 \%$ correspondiente a cenizas. Además, se identificó que el subproducto es rico en otros componentes beneficiosos para la salud, como la ergotioneina y el ergosterol, resultados mostrados en la Tabla 2. Debido a su alto contenido de proteínas, este es un buen sustrato para la preparación de proteínas hidrolizadas, obteniendo una proteína de mejor calidad, ya que se pueden obtener compuestos como péptidos y aminoácidos de interés industrial.

\section{Table 2}

Composición de la materia prima.

Componente
Proteina
Carbohidratos
Grasa cruda
Fibra
Materia seca
Ceniza
Ergotioneina
Polifenoles
Ergosterol

Cantidad porcentual
$28.6 \pm 1.18 \%$
$59.4 \pm 3.21 \%$
$3.52 \pm 0.25 \%$
$17.5 \pm 1.12 \%$
$7.3 \pm 1.2 \%$
$92.7 \pm 0.9 \%$
$8.83 \pm 0.29 \%$
$0.22 \pm 0.02 \mathrm{mg} \mathrm{ERG} / \mathrm{g}$
$3.9 \pm 0.22 \mathrm{mg} \mathrm{GAE} / \mathrm{g}$
$6.42 \pm 0.15 \mathrm{mg} / \mathrm{g}$




\subsection{Hidrólisis enzimática y grado de hidrólisis de la $\mathrm{HTCH}$}

Para la hidrólisis enzimática, se usó 4 diferentes proteasas para determinar cuál de ellas es la que tiene los mejores resultados, todas bajo las mismas condiciones de control como son $\mathrm{pH}$, temperatura, agitación y concentración de enzimas. Las enzimas que se utilizaron fueron Alcalasa ${ }^{\circledR}$, Flavourzyme ${ }^{\circledR}$, Papaína y Bioproteasa LA-450 enzimas utilizadas en la industria alimentaria. Para volúmenes menores a 2 L, se usó el esquema de hidrólisis como se indica en la Figura 1 y para volúmenes más grandes, se usó un reactor automatizado de $4 \mathrm{~L}$ y siguiendo las mismas condiciones de control de $\mathrm{pH}$, temperatura, agitación y relación E/S. Los resultados se muestran en la Figura 2, donde se puede observar tanto el volumen de $\mathrm{NaOH}$ consumido como el $\mathrm{GH}$ obtenido de los hidrolizados. Las hidrólisis más eficientes es decir las que conducen a un mayor $\mathrm{GH}$ es cuando se utilizan la Bioproteasa LA-450 y la Alcalasa ${ }^{\circledR}(6.65 \pm 0.6$ y $6.21 \pm$ 0.9) respectivamente, ya que durante la hidrólisis se consume más cantidad de base $(\mathrm{NaOH})$, esto debido a que la mayor cantidad de aminoácidos y péptidos se hidrolizan.

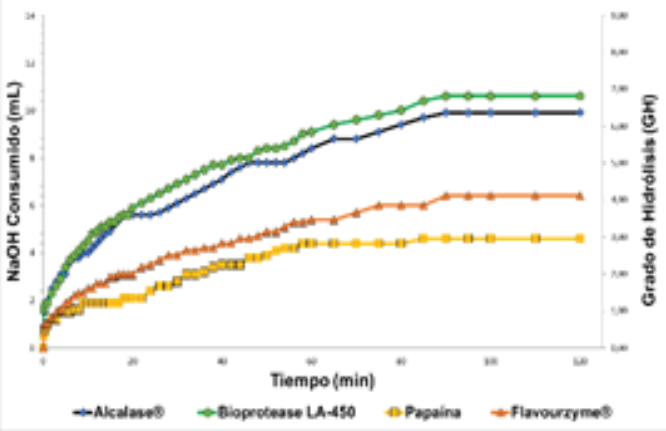

Figure 2

Curva de hidrolizados enzimáticos

Para el cálculo del GH se usó la ecuación de Aldler-Nissen y se usó 5.45 como factor de proteína para los hongos. El GH indica la escisión de los enlaces peptídicos y también influye en el rendimiento del producto, la recuperación de proteínas, las propiedades funcionales y la calidad organoléptica de los hidrolizados. El GH aumentó apreciablemente $(P \leq 0.05)$ dentro de las $2 \mathrm{hr}$ de hidrólisis y $10 \%(\mathrm{p} / \mathrm{v})$ de la concentración de sustrato, como se puede observar en la Figura 2. A partir de estos resultados, se eligió el tiempo de $2 \mathrm{hr}$ como el tiempo óptimo para la generación de hidrolizados de proteínas en los sustratos de hongos comestibles.

Como puede observarse en la Tabla 3 las enzimas Alcalasa ${ }^{\circledR}$ y Bioproteasa LA-450 son las que mayor cantidad de productos solubilizan ( $41.7 \pm 3 \%$ y $46.3 \pm 3.6 \%$ ), mientras que la enzima de origen vegetal (papaína) presenta un rendimiento más bajo. Además, se obtuvo una menor cantidad de precipitado $(13.8 \pm 1.0 \mathrm{~g})$ y un mayor volumen de sobrenadante $(13.9 \pm 1.1 \mathrm{~g})$ para el caso de la Bioproteasa LA-450, lo que indica que hay más cantidad de productos solubles. 
Table 3

Balance de masas, referido al peso seco recuperado en el precipitado y en el sobrenadante, tras la hidrólisis enzimática simple de la HTCH.

\begin{tabular}{|c|c|c|c|c|}
\hline Enzima & Alcalasa $^{\circledR}$ & Bioproteasa LA-450 & Papaína & Flavourzyme ${ }^{\circledR}$ \\
\hline $\mathrm{HTCH}(\mathrm{g})$ & 30 & 30 & 30 & 30 \\
\hline Precipitado (g) & $16.2 \pm 1.3$ & $13.8 \pm 1.0$ & $19.3 \pm 2.2$ & $20.1 \pm 1.7$ \\
\hline Sobrenadante (g) & $12.5 \pm 0.9$ & $13.9 \pm 1.1$ & $8.0 \pm 0.7$ & $6.9 \pm 0.5$ \\
\hline Rendimiento (\%) & $41.7 \pm 3.0$ & $46.3 \pm 3.6$ & $26.3 \pm 2.3$ & $23.0 \pm 1.7$ \\
\hline Proteína Hidrolizada (\%) & $76.3 \pm 3.1$ & $77.2 \pm 2.7$ & $49.4 \pm 2.0$ & $41.3 \pm 1.6$ \\
\hline
\end{tabular}

\subsection{Análisis químico del hidrolizado}

El análisis químico del hidrolizado con respecto al sustrato, como se puede ver en la Tabla 4.

Table 4

Análisis químico del hidrolizado.

\begin{tabular}{llllll}
\hline Hidrolizado & p.s. & Cenizas & M.O. & Pt & HC $_{\text {totales }}$ \\
HTCH $_{H 2 O}$ & $8.8 \pm 0.4$ & $7.6 \pm 0.3$ & $92.4 \pm 0.3$ & $25.7 \pm 1.7$ & $67.1 \pm 4.3$ \\
\hline $\mathbf{H P T C H}_{A}$ & $41.7 \pm 3.0$ & $9.2 \pm 0.6$ & $90.8 \pm 0.6$ & $51.3 \pm 3.9$ & $32.5 \pm 3.8$ \\
HPTCH $_{L A 450}$ & $46.3 \pm 3.6$ & $9.4 \pm 0.3$ & $90.6 \pm 0.3$ & $51.9 \pm 4.8$ & $41.1 \pm 4.1$ \\
HPTCH $_{P}$ & $26.3 \pm 2.3$ & $8.9 \pm 0.9$ & $91.1 \pm 0.9$ & $32.8 \pm 3.0$ & $58.1 \pm 3.2$ \\
HPTCH $_{F}$ & $23.0 \pm 1.7$ & $9.1 \pm 0.8$ & $90.9 \pm 0.8$ & $38.8 \pm 2.1$ & $54.4 \pm 4.4$
\end{tabular}

$\mathbf{H T C H}_{H 20}$ : Extracto acuoso de $\mathrm{HTCH} ; \mathbf{H P T C H}_{A}$ : Hidrolizado de $\mathrm{HCTH}$ con Alcalasa ${ }^{\circledR} ; \mathbf{H P T C H}_{L A 450}$ : Hidrolizado de HCTH con Bioproteasa LA-450; $\mathbf{H P T C H}_{p}$ : Hidrolizado de HCTH con Papaína; $\mathbf{H P T C H}_{F}$ : Hidrolizado de HCTH con Flavourzyme ${ }^{\circledR}$; p.s.: peso seco; M.O.: Materia orgánica; Pt: Proteínas Totales; HC: Hidratos de carbono; Valores expresado en Porcentaje (\%) de p.s.

Como se puede observar $\mathrm{HPTCH}_{L A 450}$ presenta mayor contenido de proteínas totales $(51.9 \pm 4.8)$ frente $a \mathrm{HTCH}_{H 2 O}(25.7 \pm 1.7)$, lo que indica que exite más producto solubilizado.

\subsection{Caracterización electroforética de los hidrolizados}

Este proceso se realizó con el fin de obtener información sobre el tamaño de los péptidos presentes en los hidrolizados. Se tiñó los geles con azul de Coomassie donde no se observó resultados por lo que se optó por la tinción de plata con ligeras modificaciones.

Como podemos observar en la Figura 3 en el carril-1 no se observan proteínas debido a que la tinción de plata no permite detectar proteínas grandes. Sin embargo, en el carril-2 se observa claramente una banda de unos $30 \mathrm{kDa}$, correspondiente a un fragmento proteico de ese tamaño, además se observa una banda intensa, de unos 5 $\mathrm{kDa}$, que corre con el frente de la electroforesis, correspondiente a péptidos de este tamaño. Análogamente, en el carril-3 se observan claramente 2 bandas, una de 27 kDa 


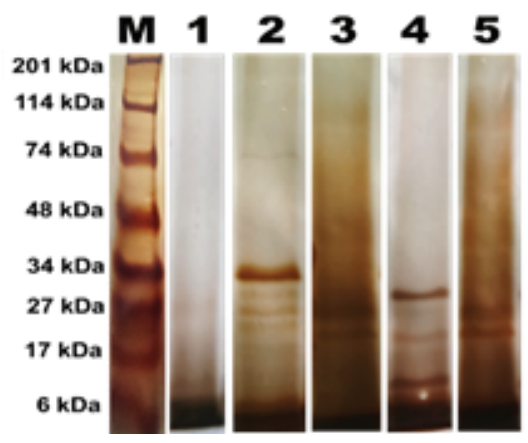

Figure 3

Gel de electroforesis en geles para péptidos, (según Schäger) de los diferentes hidrolizados proteicos y del extracto acuoso de la HTCH. (M: marcadores de peso molecular; 1: $\mathrm{HTCH}_{H 2 O}$; 2: $\mathrm{HPTCH}_{A}$; 3: $\mathrm{HPTCH}_{P}$; 4: $\mathrm{HPTCH}_{L A 450}$; 5: $\mathrm{HPTCH}_{F}$.)

y otra de $20 \mathrm{kDa}$, correspondientes a fragmentos proteicos liberados y solubilizados por la Papaína. En el carril-4 se logran distinguir tres bandas de 29 kDa, 20 kDa y 10 kDa que corresponderían a fragmentos de proteínas con este peso molecular. En el carril-5, se observa tenuemente dos bandas, una banda de $41 \mathrm{kDa}$, y una de $27 \mathrm{kDa}$ también se observa otra banda intensa de 17 kDa que correspondería a péptidos de ese tamaño.

\subsection{Caracterización del hidrolizado por cromatografía de exclusión}

El propósito de este procedimiento es separar los péptidos de acuerdo a su tamaño, y la distribución del tamaño de las proteínas, péptidos, oligopéptidos y aminoácidos presentes en los hidrolizados. Los resultados obtenidos se muestran en la Figura 4 , correspondiente al cromatograma obtenido a $280 \mathrm{~nm}$, para cada uno de los hidrolizados.

Como se puede observar el cromatograma se ha dividido en tres grandes zonas: $>5 \mathrm{kDa},<5 \mathrm{kDa}$ y $>0.2 \mathrm{kDa}, \mathrm{y}<0.2 \mathrm{kDa}$. La zona ' $>5 \mathrm{kDa}$ ', constituida por proteínas y péptidos mayores de $5 \mathrm{kDa}$; la zona ' $<5 \mathrm{kDa}$ y $>0.2 \mathrm{kDa}$ ', constituida por péptidos y oligopéptidos menores de $5 \mathrm{kDa}$, y mayores de $0.2 \mathrm{kDa}$, y la zona ' $<0.2 \mathrm{kDa}$ ' constituida por dipéptidos y aminoácidos libres.

En la Tabla 5 se muestra la contribución porcentual de cada zona o área, respecto del total, para cada hidrolizado, donde los mayores porcentajes de dipéptidos y aminoácidos libres se encuentran para $\mathbf{H P T C H} \mathbf{H}_{F}$, lo que podría atribuirse a las exoproteasas presentes.

Desde un punto de vista comercial debido al costo y al uso final de los hidrolizados, no es conveniente aislar los péptidos, sino solo se purificarán y/o concentrarán por medio de membranas. 

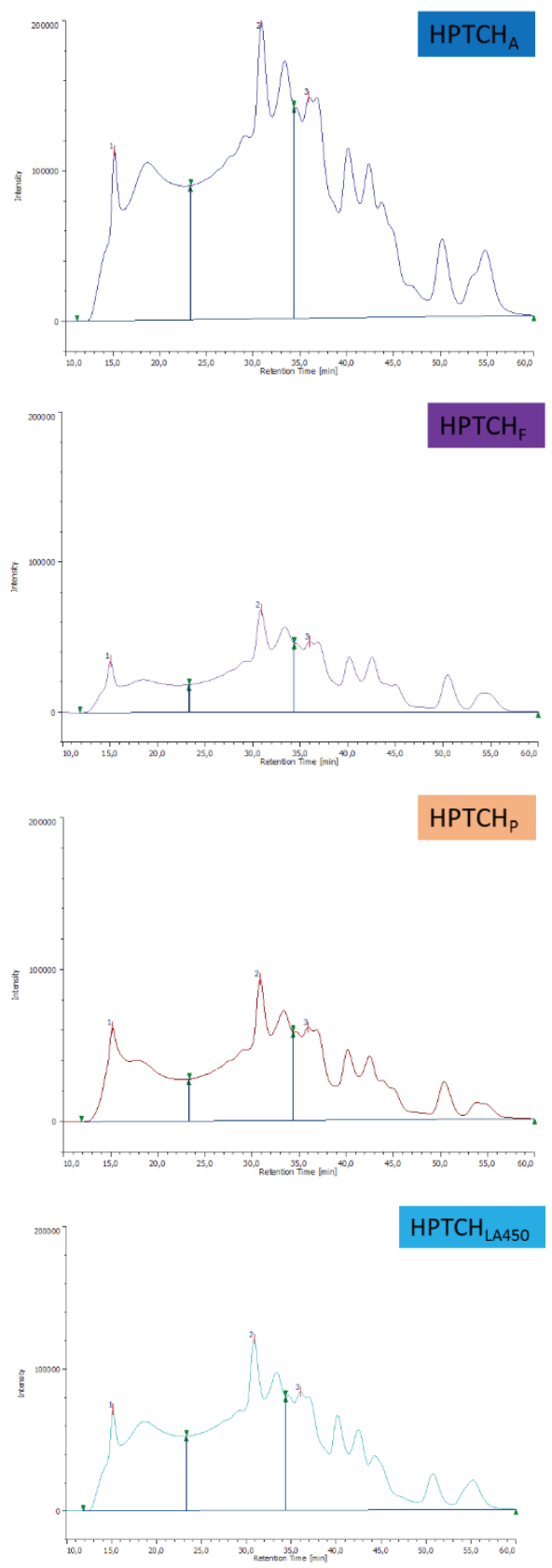

Figure 4

Caracterización de los hidrolizados enzimáticos por Cromatografía de Exclusión (280 nm). 1: $\mathrm{HPTCH}_{A}$; 2: $\mathrm{HPTCH}_{F}$; 3: $\mathrm{HPTCH}_{P} ;$ 4: $\mathrm{HPTCH}_{L A 450}$

\section{Discusiones}

Del análisis aproximado del subproducto se logró identificar que en su composición contiene varios productos de interés comercial, estos resultados coincidieron con los estudios informados anteriormente [2, 25]. La especificidad de las enzimas es un elemento primario que influye en las características de los hidrolizados, es decir, la composición y las secuencias de aminoácidos de los péptidos producidos dependerán 
Table 5

Contribución porcentual para cada una de las zonas seleccionadas en los cromatogramas $(280 \mathrm{~nm})$.

$280 \mathbf{n m}$
$\mathrm{HTCH}_{H 2 O}$
$\mathrm{HPTCH}_{A}$
$\mathrm{HPTCH}_{L A 450}$
$\mathrm{HPTCH}_{P}$
$\mathrm{HPTCH}_{F}$

$>5 k$ Da (\%)
6.68
23.71
25.13
24.58
19.05

$<5->0.2 \mathrm{kDa}(\%)$
30.19
38.35
38.88
38.58
38.92

$<0.2$ kDa (\%)
63.12
37.92
35.98
36.83
42.02

en gran medida del tipo de proteasa y sustrato que se haya utilizado [10, 11, 26]. El aumento del GH estuvo de acuerdo con informes previos de hidrólisis de proteínas donde $\mathrm{GH}$ se mejoró con el aumento de las cantidades de enzimas [27-29], esto indica el porcentaje de enlaces peptídicos rotos en relación con la proteína original. Tradicionalmente, las proteasas microbianas, especialmente las proteasas alcalinas, se han utilizado en la preparación de hidrolizados de proteínas de alto valor. Los hidrolizados tienen una variedad de aplicaciones y pueden usarse en formulaciones de alimentos, productos alimenticios terapéuticos específicos, fortificación de jugos de frutas y refrescos, como aditivos alimentarios funcionales, alimentación animal, etc. $[26,30]$.

\section{Conclusiones}

Estos resultados permitieron determinar que la Bioproteasa LA-450 utilizada como enzima para el proceso de hidrólisis de proteínas es la más eficiente, además que los hidrolizados de proteínas son un buen procedimiento para mejorar la calidad de la proteína nativa y a su vez poder obtener mayor cantidad de aminoácidos y péptidos que pueden usarse a nivel industrial tanto en la industria cosmética, Médico farmacéutica, y Agronomía. Adicionamente podrian usarse como un medio de fermentación para la producción de hidrolasas, particularmente quitinasas.

\section{Agradecimientos}

Agradecemos al Ministerio de Ciencia español e Innovación para el apoyo financiero de este trabajo (Proyecto RTC2015-4039-2), que cuenta con apoyo financiero parcial del FEDER fondos de la Unión Europea. A la Universidad Técnica de Manabí por permitirnos colaborar con ellos.

\section{Conflicto de Intereses}

Declaro que no existen intereses particulares por parte de los autores que pudiesen afectar directa o indirectamente a los resultados. 


\section{References}

[1] Royse et al. Edible and medicinal mushrooms: Technology and applications. Cunha ZD and Arturo PG, editors. Wiley Online Library; 2017. Chapter

[2] Cremades O, Diaz-Herrero MM, Carbonero-Aguilar P, Gutierrez-Gil JF, Fontiveros E, Bautista J. White button mushroom ergothioneine aqueous extracts obtained by the application of enzymes and membrane technology. Food Biosci. 2015;10:42-47.

[3] Bishop KS, Kao CH, Xu Y, Glucina MP, Paterson RRM, Ferguson LR. From 2000 years of Ganoderma lucidum to recent developments in nutraceuticals. Phytochemistry. 2015;114:56-65

[4] Amza T, Balla A, Tounkara F, Man L, Zhou HM. Effect of hydrolysis time on nutritional, functional and antioxidant properties of protein hydrolysates prepared from gingerbread plum (Neocarya macrophylla) seeds. Int Food Res J. 2013;20(5):2081-2090.

[5] Kristinsson HG, Rasco BA. Fish protein hydrolysates: production, biochemical, and functional properties. Crit Rev Food Sci Nutr. 2013;40(1):43-81.

[6] Aspmo SI, Horn SJ, Eijsink VG. Enzymatic hydrolysis of Atlantic cod (Gadus morhua L.) viscera. Process Biochem. 2005;40(5):1957-1966.

[7] Darewicz M, Borawska J, Vegarud GE, Minkiewicz P Iwaniak A. Angiotensin I-converting enzyme (ACE) inhibitory activity and ACE inhibitory peptides of salmon (Salmo salar) protein hydrolysates obtained by human and porcine gastrointestinal enzymes. Int J Mol Sci. 2014;15(8):14077-14101.

[8] Silveira ST, Martínez-Maqueda D, Recio I, Hernández-Ledesma B. Dipeptidyl peptidase-IV inhibitory peptides generated by tryptic hydrolysis of a whey protein concentrate rich in $\beta$-lactoglobulin. Food Chem X. 2013;141(2):1072-1077.

[9] Millward DJ, Layman DK, Tomé D, Schaafsma G. Protein quality assessment: impact of expanding understanding of protein and amino acid needs for optimal health. Am. J. Clin. Nutr. 2008;87(5):1576S$1581 S$.

[10] Mccarthy AL, O'Callaghan YC, O'Brien NM. Protein hydrolysates from agricultural crops-bioactivity and potential for functional food development. Agriculture. 2013; 3(1):112-130.

[11] Xu X, Guo S, Hao X, Ma H, Bai Y, Huang Y. Improving antioxidant and antiproliferative activities of colla corii asini hydrolysates using ginkgo biloba extracts. Food Sci Nutr. 2018. doi: 10.1002 / fsn3.587

[12] Farvin KHS, Andersen LL, Otte J, Nielsen HH, Jessen F, Jacobsen C. Antioxidant activity of cod (Gadus morhua) protein hydrolysates: Fractionation and characterisation of peptide fractions. Food Chem. 2016;204:409.

[13] Karnjanapratum S, O'Callaghan YC, Benjakul S, O'Brien N. Antioxidant, immunomodulatory and antiproliferative effects of gelatin hydrolysate from unicorn leatherjacket skin. J. Sci. Food Agric. 2015;96:3220-3226.

[14] Sah BNP, Vasiljevic T, Mckechnie S, Donkor ON. Effect of probiotics on antioxidant and antimutagenic activities of crude peptide extract from yogurt. Food Chem. 2014;156:264

[15] Inca-Torres AR, Urbina-Salazar ADR, Falcón-García G et al. Hydrolytic enzymes production by Bacillus licheniformis growth on fermentation media formulated with sewage sludge. J Biotech Res. 2018;9:1426.

[16] AOAC. $18^{\text {th }}$ ed. Washington, D.C.: Official Methods of Analysis; 2006

[17] MAPA. Madrid: Métodos oficiales de análisis; 1994.

[18] Inca-Torres A R. Revalorización de los subproductos de la industria de hongos y setas comestibles. Aplicación a la obtención de productos de alto valor añadido. [Doctoral thesis]. Sevilla: Universidad de Sevilla; 2019

[19] Bagul MB, Sonawane SK, Arya SS. Bioactive characteristics and optimization of tamarind seed protein hydrolysate for antioxidant-rich food formulations. 3 Biotech. 2018;8(4):218.

[20] Sewczyk T, Antink MH, Maas M, Kroll S, Beutel S. Flow rate dependent continuous hydrolysis of protein isolates. AMB Express. 2018;8(1):18

[21] Espejo CFJ. Obtención de hidrolizados de proteínas de leche de cabra con actividad inhibidora de la enzima convertidora de la angiotensina. [Doctoral thesis]. Granada: Universidad de Granada; 2013.

[22] Adler-Nissen J. Enzymatic hydrolysis of food proteins. Elsevier; 1986.

[23] Laemmli UK. Cleavage of structural proteins during the assembly of the head of bacteriophage T4. Nature. 1970;227:680-685.

[24] Schägger H, Von JG. Tricine sodium dodecyl sulfate polyacrylamide gel electrophoresis for protein separation in the range 1 to $100 \mathrm{kDa}$. Anal. Biochem. 1987;166(2):368-379.

[25] Cremades O, Diaz-Herrero MM, Carbonero-Aguilar $P$ et al. Preparation and characterisation of seleniumenriched mushroom aqueous enzymatic extracts (MAEE) obtained from the white button mushroom (Agaricus bisporus). Food Chem. 2012;133(4):1538-1543. 
[26] Chalamaiah M, Kumar BD, Hemalatha R, Jyothirmayi T. Fish protein hydrolysates: Proximate composition, amino acid composition, antioxidant activities and applications: A review. Food Chem. 2012;135:3020-3038.

[27] Hou H, Li B, Zhao X, Zhang Z, Li P. Optimization of enzymatic hydrolysis of Alaska pollock frame for preparing protein hydrolysates with low-bitterness. LWT. Food Sci. Technol. Res. 2011;44(2):421-428.

[28] Villanueva A, Clemente A, Bautista J, Millán F. Production of an extensive sunflower protein hydrolysate by sequential hydrolysis with endo- and exo-proteases. Grasas y Aceites. 1999a;50(6):472-476.

[29] Villanueva A, Vioque J, Sánchez-Vioque $R$ et al. Peptide characteristics of sunflower protein hydrolysates. J Am Oil Chem Soc. 1999b;76(12):1455-1460.

[30] Ward AJ, Hobbs PJ, Holliman PJ, Jones DL. Optimisation of the anaerobic digestion of agricultural resources. Bioresour. Technol. 2008;99(17):7928-7940. 